\title{
Remote migration of breast filler to the inguinal area: a case report
}

\section{Jangyoun Choi, Ye Sol Kim, Deuk Young Oh}

Department of Plastic and Reconstructive Surgery, Seoul St. Mary's Hospital, College of Medicine, The Catholic University of Korea, Seoul, Korea

This article was presented at the Aesthetic Plastic Surgery on July 11, 2021.
Polyacrylamide hydrogel (PAAG) is an injectable material for breast augmentation that was developed in the 1980s. It was widely used until the 2000s, but subsequently lost its popularity due to high complication rates. In this case report, we share our experience of a patient complaining of hard and asymmetric breasts who had received PAAG injections 7 years ago. Surprisingly, we found that the filler in the left breast had migrated to the left inguinal area. Although breast fillers have lost ground in recent years, we think that long-term complications of fillers, as observed in this case, will still arise in the future. Therefore, young plastic surgeons who do not have direct experience with this material should be aware of its possible complications.

Keywords Polyacrylamide gel / Dermal fillers / Foreign-body migration / Mammaplasty

\section{INTRODUCTION}

Polyacrylamide hydrogel (PAAG) is a polymer composed of polyacrylamide and water. Introduced in the early 1990s, it was widely advertised for its nontoxicity, non-resorbability, and biocompatibility [1]. First used as a dermal filler to improve skin contour, its use was expanded to breast augmentation. Over time, it lost popularity and was banned in some nations due to various complications [2]. Complications include but are not limited to, indurations, infection, breast pain, contracture, and migration. Local migration is common, but migration to a distant location is relatively rare [3]. In this case report, we share a case of PAAG migration that was found outside the regional anatomy. Its presentation, diagnostic challenge, and management are presented.

Received: Jul 26, 2021 Revised: Aug 27, 2021 Accepted: Aug 31, 2021 Correspondence: Deuk Young Oh Department of Plastic and Reconstructive Surgery, Seoul St. Mary's Hospital, College of Medicine, The Catholic University of Korea, 222 Banpo-daero, Seocho-gu, Seoul 06591, Korea Tel: +82-2-2258-2841, Fax: +82-2-594-7230

E-mail: ohdeuk1234@hanmail.net

Copyright @ 2021 The Korean Society for Aesthetic Plastic Surgery.

This is an Open Access article distributed under the terms of the Creative Commons Attribution Non-Commercial License (https://creativecommons.org/licenses/by-nc/4.0/) which permits unrestricted non-commercial use, distribution, and reproduction in any medium, provided the original work is properly cited. $\quad w w w . e-a a p s . o r g$

\section{CASE REPORT}

A 37-year-old patient visited our institution complaining of gradual-onset breast asymmetry. She had received polyacrylamide gel injection for breast augmentation 7 years ago.

On physical examination, both breasts were soft without erythema or induration. No signs of contracture or nipple retraction were found. The patient did not complain of breast pain. However, she stated that only her left breast had become smaller over the years (Fig. 1). Concurrently, she complained of gradual enlargement of her left inguinal area.

Computed tomography (CT) scans of the chest and inguinal area were undertaken. Chest CT showed a $12.3-\mathrm{cm}$ fluid collection in the right breast, suggesting filler injection. On the contrary, the expected filler material was unidentifiable on the left side (Fig. 2A). Unexpectedly, a collection of the same signal intensity was found in the inguinal and lower abdominal area, around where the patient complained of a bulge (Fig. 2B). Under the impression of foreign body reaction and distant migration of the breast filler, removal was planned for the right breast and left the inguinal area, where foreign bodies were found in CT images.

Under general anesthesia, right inframammary and left inguinal crease incisions were made. Upon dissection down to the subglandular plane, a fibrous capsule was visible. Through the opening of the capsule, we observed a copious amount of yellow, creamy foreign body material, which was presumed to be the previously in- 




Fig. 1. Patient at initial presentation. Left-sided hypomastia and a concurrent bulge in the left inguinal area (yellow arrow) are noted. jected PAAG (Fig. 3). Although most of the material was in the subglandular plane, some portions of the foreign body showed irregular extension into the glandular tissue. The fluidic portions of the foreign body were easily removed with suction and manual squeezing.

Next, we removed the foreign body in the left inguinal area. Upon incision and dissection, the same material as in the right breast was visible, with most of it residing between the external and internal oblique muscles.

Multiple rounds of irrigation, curettage, and debridement were done in the inguinal area and breast pocket. Most portions of unhealthy granulation and gelatinous foreign body were removed. Lastly, we employed a triple-antibiotic solution (a mixture of cefazolin [2 g], metronidazole [500 mg], and gentamicin [80 mg]) to irrigate both pockets. Negative suction drains and layered closure were done. Upon histology, foreign body reactions with multiple giant cells in a gel pool were observed (Fig. 4). The tissue culture did not contain any microorganisms. The patient has been followed up for 1 year without complications.

\section{DISCUSSION}

Injectable materials for breast augmentation have a long history of initial approval, only to be prohibited subsequently based on postmarket surveillance or complication reports [4]. For example, various PAAG fillers (e.g., Aquamid, Amazingel, Agriform, Interfall,
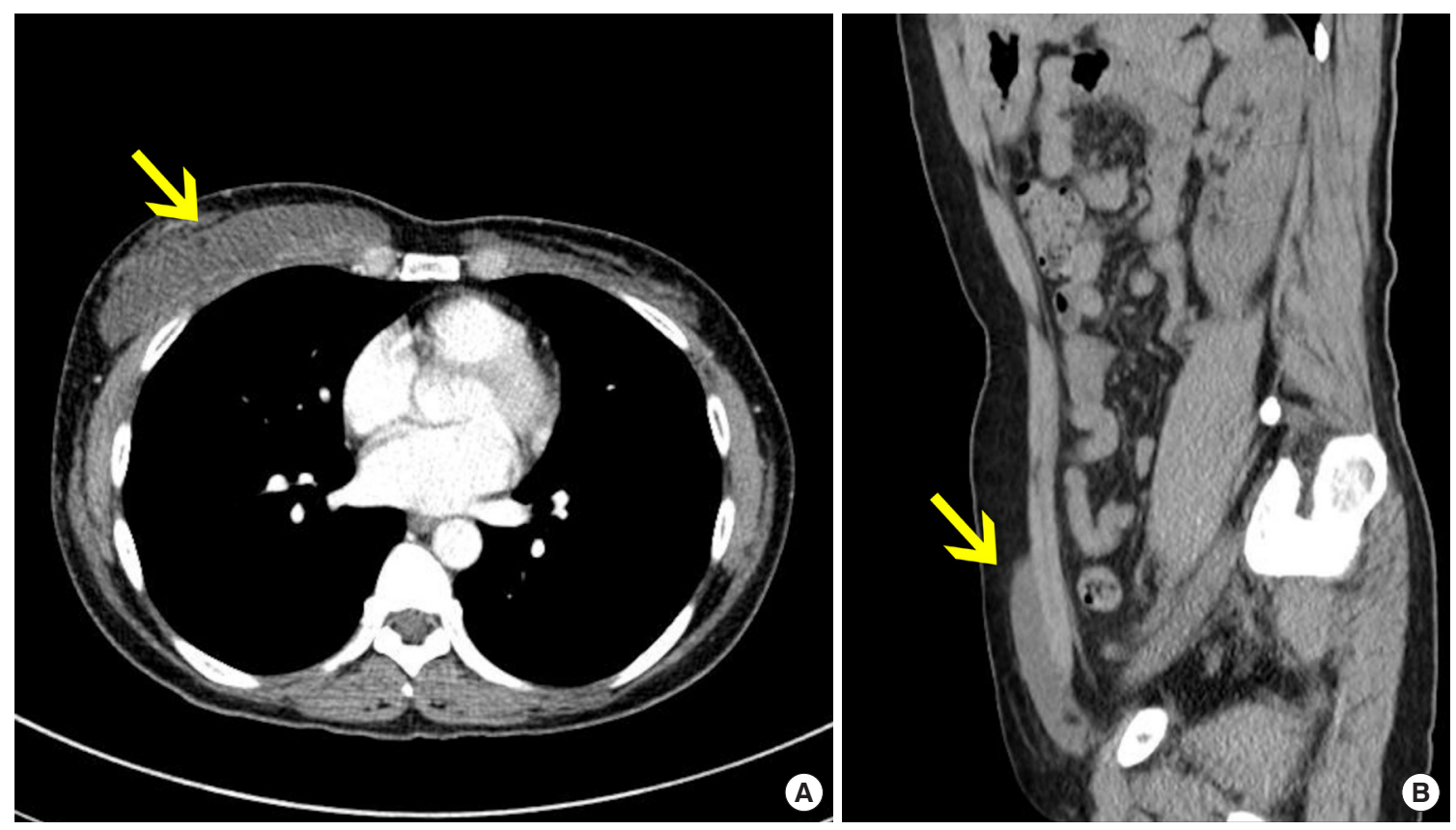

Fig. 2. Radiologic findings. (A) A 12.3-cm foreign body was present in the right retroglandular plane (yellow arrow). However, the foreign body was not as evident in the left breast. (B) Foreign body of similar attenuation as in (A), dispersed between the external and internal oblique muscles, mostly accumulated caudally in the inguinal area (yellow arrow). 

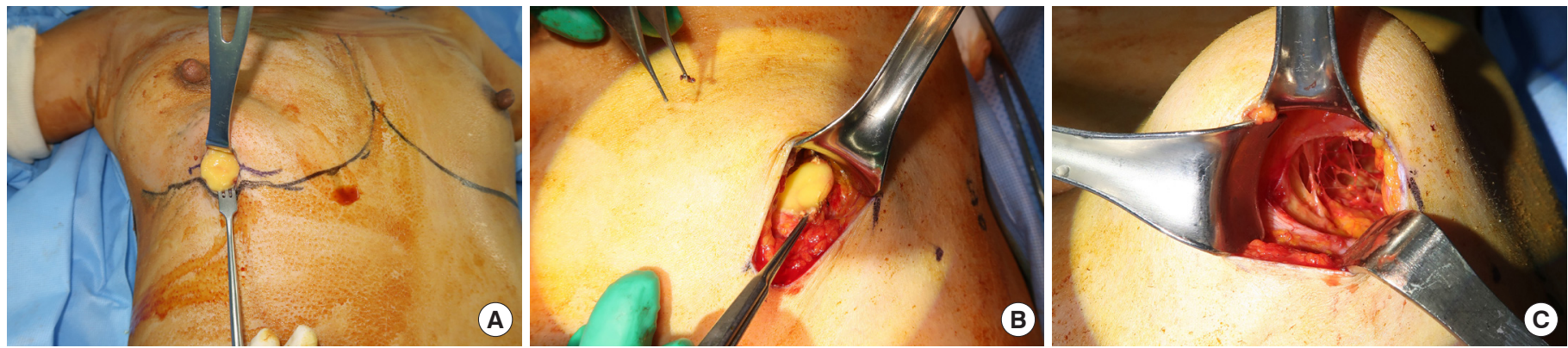

Fig. 3. Intraoperative findings. (A) A pool of a yellow, creamy foreign body was seen in the retromammary space. This material could be readily evacuated due to its gelatinous character. (B) The same material was found in the left inguinal area. (C) Despite numerous vigorous rounds of excision and debridement, film-like stains of the foreign body around the normal tissue still remained.
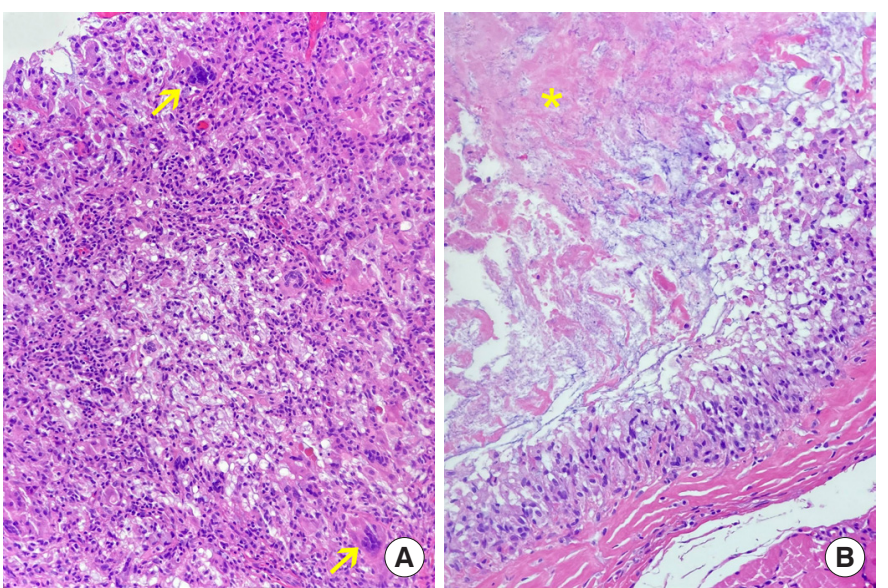

Fig. 4. Histology of the removed foreign body and adjacent tissue. (A) A dense accumulation of inflammatory cells and multinucleated giant cells (yellow arrows) $(H \& E, \times 100)$. (B) Collection of gel pooled under the dermal layer (yellow asterisk) (H\&E, × 200).

Formacryl, and Bioformacryl) are commercially available, but have been reported to show high complication rates [5]. Consequently, regulatory approval has been withdrawn in some nations, including China and Eastern European countries [6,7].

Although it seems that a consensus has been formed regarding the material's long-term behavior, active discussion and reports can still be seen in recent publications [8-10]. One of the largest investigations analyzed 325 cases of PAAG complications [11]. The interval between PAAG injection and the onset of adverse reactions ranged from 18 days to 20 years. The single most common complication was breast pain (45.54\%), followed by induration and deformation. Almost half (47.69\%) of the patient cohort complained of multiple symptoms. Treatment of the complications was often possible with evacuation alone (32\%), but most cases required some degree of partial mastectomy (51\%). Ninety-two percent of the cohort reported improvement of the symptoms, but about 5\% needed reoperation. Fortunately, evacuation without mastectomy was sufficient to relieve our patient's symptoms.

Another study analyzed 10 years of experience regarding surgi- cal treatment of PAAG-related complications [6]. The authors devised a classification system (type I: pure subglandular, type II: scattered, type III: migration) according to the anatomical distribution of complications. They also suggested a treatment algorithm based on their classification system, which allows a thorough investigation of related complications. This algorithm also aids in the selection of appropriate surgical methods, as well as the timing of secondary reconstruction. According to this algorithm, our patient corresponded to a type II (right side) and III (left side) case. We opted for open-incision surgery through an inframammary fold approach. Since the patient did not want further augmentation, we did not proceed to secondary augmentation. Immediate augmentation would have been possible if the patient had requested.

Adding to previous reports describing the difficulty of completely evacuating PAAG, we suggest two surgical adjuncts. First, utilizing mechanical debriders is beneficial. Since PAAG shows a yellowish discoloration, visual discrimination of PAAG from the surrounding fat tissue is inaccurate. After manual drainage and suction, using instruments such as an ultrasonic or hydrosurgical debrider ensures complete surface debridement. Second, we recommend antibiotic irrigation before closure. We used combination of cephalosporin, metronidazole, and an aminoglycoside, which is a regimen widely used for breast implant salvage.

Although rare, the most alarming complication related to PAAG is malignancy. Chen et al. [12] reported two cases of malignancy in PAAG-injected patients. Messenger RNA upregulation of the proto-oncogene $c-m y c$ was identified in vitro, suggesting the carcinogenic and cytotoxic potential of PAAG [13]. For this reason, we suggest that PAAG-injected patients need intensive follow-up, not only for surgical complications, but also for cancer surveillance.

Except for autologous fat grafting, experts now advise against using injectables for breast augmentation [2]. Although injectables have fallen out of favor in most practices, their long-term consequences will still be seen in clinics. This report reiterates the serious consequences of injectables. 


\section{NOTES}

\section{Conflicts of Interest}

No potential conflict of interest relevant to this article was reported.

\section{Ethical Approval}

The study was performed in accordance with the principles of the Declaration of Helsinki.

\section{Patient Consent}

The patient provided written informed consent for the publication and the use of her images.

\section{ORCID}

Jangyoun Choi

Ye Sol Kim

Deuk Young Oh

https://orcid.org/0000-0002-5165-8414

https://orcid.org/0000-0003-3970-9212

https://orcid.org/0000-0003-3499-1554

\section{REFERENCES}

1. Yang Y, Li S, He J, et al. Clinicopathological analysis of 90 cases of polyacrylamide hydrogel injection for breast augmentation including 2 cases followed by breast cancer. Breast Care (Basel) 2020;15:38-43.

2. Winter J, Shiga S, Islur A. The complications of polyacrylamide hydrogel augmentation mammoplasty: a case report and review of the literature. Plast Surg Case Studies 2016;2:47-50.

3. Zhang MX, Li SY, Xu LL, et al. Repeated lumps and infections: a case report on breast augmentation complications. World J Clin Cases 2019; $7: 3322-8$
4. Kontis TC, Rivkin A. The history of injectable facial fillers. Facial Plast Surg 2009;25:67-72.

5. Rish A. Polyacrylamide gel 10 years experience: with particular reference to complications from filling of the body of the lip. J Cosmet Dermatol 2014;13:253-60.

6. Jin R, Luo X, Wang X, et al. Complications and treatment strategy after breast augmentation by polyacrylamide hydrogel injection: summary of 10-year clinical experience. Aesthetic Plast Surg 2018;42:402-9.

7. Cheng NX, Wang YL, Wang JH, et al. Complications of breast augmentation with injected hydrophilic polyacrylamide gel. Aesthetic Plast Surg 2002;26:375-82.

8. Kim HJ, Lee SJ, Lee JH, et al. Breast reconstruction after complications following breast augmentation with massive filler injections. Medicine (Baltimore) 2020;99:e21516.

9. Ku I, Park JU. Complications of polyacrylamide hydrogel injection for breast augmentation: a case report and literature review. Arch Aesthetic Plast Surg 2019;25:119-23.

10. Mo YW, Lee DL, Shin HK, et al. Polyacrylamide hydrogel (Aquamid) filler removal after a decade. Arch Aesthetic Plast Surg 2019;25:115-8.

11. Qian B, Xiong L, Guo K, et al. Comprehensive management of breast augmentation with polyacrylamide hydrogel injection based on 15 years of experience: a report on 325 cases. Ann Transl Med 2020;8:475.

12. Chen G, Wang Y, Huang JL. Breast cancer following polyacrylamide hydrogel injection for breast augmentation: a case report. Mol Clin Oncol 2016;4:433-5.

13. Xi TF, Fan CX, Feng XM, et al. Cytotoxicity and altered c-myc gene expression by medical polyacrylamide hydrogel. J Biomed Mater Res A 2006;78:283-90. 